\title{
Population Pharmacokinetics of Hydroxychloroquine in COVID-19 Patients: Implications for Dose Optimization
}

\author{
Pauline Thémans ${ }^{1} \cdot$ Leila Belkhir $^{2}$. Nicolas Dauby,4 . Jean-Cyr Yombi ${ }^{2}$. Julien De Greef ${ }^{2}$. \\ Kevin-Alexandre Delongie ${ }^{5} \cdot$ Martin Vandeputte $^{4} \cdot$ Rakan Nasreddine $^{3} \cdot$ Xavier Wittebole $^{2} \cdot$ Francoise Wuillaume $^{4}$.

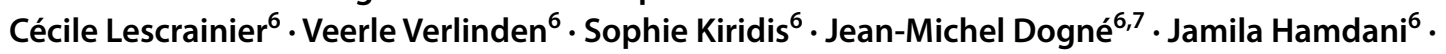 \\ Pierre Wallemacq ${ }^{5} \cdot$ Flora T. Musuamba $^{6,8}$ (1)
}

Published online: 23 September 2020

(c) Springer Nature Switzerland AG 2020

\begin{abstract}
Background and Objective In the absence of characterization on pharmacokinetics and reference concentrations for hydroxychloroquine in COVID-19 patients, the dose and treatment duration for hydrochloroquine are currently empirical, mainly based on in vitro data, and may vary across national guidelines and clinical study protocols. The aim of this paper is to describe the pharmacokinetics of hydroxychloroquine in COVID-19 patients, considered to be a key step toward its dosing optimization.

Methods We have developed a population pharmacokinetic model for hydroxychloroquine in COVID-19 patients using prospectively collected pharmacokinetic data from patients either enrolled in a clinical trial or treated with hydroxychloroquine as part of standard of care in two tertiary Belgian hospitals.

Results The final population pharmacokinetic model was a one-compartment model with first-order absorption and elimination. The estimated parameter values were $9.3 / \mathrm{h}, 860.8 \mathrm{~L}$, and $15.7 \mathrm{~L} / \mathrm{h}$ for the absorption rate constant, the central compartment volume, and the clearance, respectively. The bioavailability factor was fixed to 0.74 based on previously published models. Model validations by bootstraps, prediction corrected visual predictive checks, and normalized prediction distribution errors gave satisfactory results. Simulations were performed to compare the exposure obtained with alternative dosing regimens.

Conclusion The developed models provide useful insight for the dosing optimization of hydroxychloroquine in COVID-19 patients. The present results should be used in conjunction with exposure-efficacy and exposure-safety data to inform optimal dosing of hydroxychloroquine in COVID-19.
\end{abstract}

Pauline Thémans and Leila Belkhir have equally contributed to the manuscript.

Electronic supplementary material The online version of this article (https://doi.org/10.1007/s13318-020-00648-y) contains supplementary material, which is available to authorized users.

Flora T. Musuamba

Flora.MusuambaTshinanu@fagg-afmps.be

Extended author information available on the last page of the article 


\section{Key Points}

Inconsistent doses of hydroxychloroquine are included in national guidelines and clinical study protocols for the management of COVID-19 disease

Modeling and simulation approaches have recently been proposed for dose selection, but (external) clinical validation was either lacking or carrying important limitations and unverified assumptions

We propose a population pharmacokinetic model developed using prospectively collected hydroxychloroquine concentrations in COVID-19 patients. This model constitutes a basis for dose optimization

Simulation results showed that previously developed models in rheumatoid arthritis and lupus erythematosus over-predicted COVID-19 patient concentrations

\section{Introduction}

Hydroxychloroquine (HCQ) is a 4-aminoquinolein drug approved and used for decades for the treatment of malaria [1], rheumatoid arthritis (RA) [2], and cutaneous lupus erythematosus (CLE) [3]. During the first peak of the disease, HCQ was extensively used off-label for the management of coronavirus pandemic, as one of the drugs previously approved in other indications and being repurposed to treat COVID-19 patients. The first evidence for potential efficacy originated from in vitro experiments in kidney cells (VeroE6) that suggested that HCQ might be effective against COVID-19 [4-6]; however, the in vivo and clinical relevance of these experiments were challenged in recent publications on macaques [7]. Based on the results of in vitro studies showing antiviral activity of HCQ on SARS-CoV-2, and based on some preliminary results from small clinical studies [8,9], interim guidelines in several countries recommended treating hospitalized patients suffering from moderate to severe COVID-19 with HCQ at various dosing regimens [10]. Studies completed thus far have used different dosing regimens, and show variable and inconsistent results regarding the efficacy of HCQ in COVID-19 based on clinically relevant endpoints, such as mortality: some studies showed positive efficacy results in COVID-19 patients, whereas several others failed to demonstrate any added-value for the use of HCQ as compared to standard of care or no treatment. The overall evidence on the drug efficacy remains controversial, with conflicting results reported across trials [8, 9, 11-17], and a lack of robust data.

The safety profile of HCQ is considered relatively good at the approved dose in inflammatory diseases. However, some recent data have also suggested that the safety of HCQ in COVID-19 patients might be different from the inflammatory diseases [18] There is currently a need for the characterization of HCQ safety, and in particular cardiac safety, in COVID-19 patients. [18].

The HCQ dosing regimen still needs to be optimized in order to improve the drug benefit/risk balance, which is known to be highly dependent on the dose used.

The pharmacokinetics of HCQ have been described in inflammatory indications but not so far in COVID-19-disease. Therefore, reference pharmacokinetic exposures in COVID-19 patients are unknown and so are clinical target exposures for disease cure. The doses implemented are inconsistent across publications, national guidelines, and ongoing clinical use. There is an urgent need for HCQ pharmacokinetic characterization in COVID-19, as acknowledged in recent publications. [18-20].

Population pharmacokinetic (POPPK) modeling and simulation can be considered as the most appropriate approach to obtain relevant pharmacokinetic information during the pandemic crisis, given the ability to perform acceptable analysis from sparse or random blood samples, overcoming the need for large sampling (needed with traditional pharmacokinetics), that would be very complicated to implement in the context of an outbreak.

A population approach was used to describe the pharmacokinetics of HCQ in inflammatory diseases. Two population pharmacokinetic models have been published for HCQ in patients with rheumatoid arthritis and lupus erythematosus using whole-blood HCQ concentrations. [2, 3] Four additional models describe plasma concentrations or merged blood and plasma concentrations [1, 3, 21, 22] in healthy volunteers and patients. These models have been used to simulate COVID-19 patient exposures at different doses in a recent publication, under the unverified assumption that the pharmacokinetics of HCQ would be similar in other diseases and in COVID-19 (see, e.g., [18, 23]).

The aim of this paper is to describe HCQ pharmacokinetics in COVID-19 patients and to characterize reference pharmacokinetic exposures in this population. A model-based approach is used for this purpose. Simulations of different scenarios are performed to illustrate how the model can be used for dose optimization. 


\section{Patients and Methods}

\subsection{Patients}

The pharmacokinetic data (blood concentrations) originated from 33 patients enrolled in an open-label, single-arm study evaluating the pharmacokinetics, safety, and efficacy of HCQ in COVID-19 patients in Saint-Luc University Hospital, a tertiary hospital in Brussels (Belgium). HCQ therapy was administered as per the Belgian national protocol guidance: $400 \mathrm{mg}$ twice daily (BID) on day 1, followed by $200 \mathrm{mg}$ BID the subsequent days for a 5-day total duration of treatment. Patients received Plaquenil ${ }^{\circledR}$ tablets, each tablet having $200 \mathrm{mg}$ of racemic HCQ sulfate, equivalent to $155 \mathrm{mg}$ of racemic HCQ base. This study was conducted in order to characterize the pharmacokinetics of HCQ that can serve to optimize the dosing regimen. This study was planned to enrol 50 patients with COVID-19 infection. Pharmacokinetic assessment of HCQ required at least 1 scheduled blood sampling and 1 optional blood sampling via venipuncture or venous catheter. The first sample was an opportunistic sample drawn within the first $4 \mathrm{~h}$ after dose administration, after an accurate record of the drawing time. This sample was supplemented by a blood sample at the end of treatment or at the time of treatment interruption for safety reasons.

The protocol (Eudract 2020-001434-35) was approved by the local ethical committee, and a written informed consent was obtained from each patient taking part to the study, in compliance with the International Council for Harmonisation Good Clinical Practice Guideline.

The pharmacokinetic concentrations from the 33 patients enrolled in the study were supplemented by concentrations collected from 23 patients as part of standard of care at Saint-Luc Hospital, and at Saint-Pierre Hospital, another tertiary hospital in Brussels (Belgium).

\subsection{Analytical Method}

Determination of the whole-blood HCQ concentrations at the different time points was obtained in the laboratory of Analytical Chemistry of Saint-Luc University Hospital, Department of Clinical Chemistry, by a validated liquid chromatography-tandem mass spectrometric method, on a Waters Xevo TQ-S micro, in ESI+ mode (Waters, Milford, MA, USA). Briefly, the red blood cells were hemolyzed by methanol containing HCQ-d4, used as an internal standard. The resulting sample underwent a liquid-liquid extraction using dichloromethane/ether/hexane $(30 / 50 / 20)+0.5 \%$ methylbutan-1-ol, at a basic $\mathrm{pH}$. The next step was the evaporation of the organic phase followed by the reconstitution of the dry residue in the mobile phase. The reconstituted sample was injected into a BEHC18 UPLC column, $2.1 \times 100$,
$1.7 \mu \mathrm{m} f$ (Waters). The mobile phase consisted of a buffer, ammonium formate $5 \mathrm{mM}$, at $\mathrm{pH} 3$ with acetonitrile $+0.1 \%$ formic acid. The method was linear over the concentration range of $100-3000 \mathrm{ng} / \mathrm{mL}$. Between-day precision measured using 3 internal quality control samples were all within $10 \%$. Accuracy results were also very satisfactory with a bias of $<0.3 \%$ obtained with external quality control sample.

\subsection{POPPK Model Development and Validation}

Data were analyzed using nonlinear mixed effects modeling in NONMEM software, v.7.3 (Icon Development Solutions, Ellicott City, MD, USA). Model parameters were estimated based on data collected during the study (33 patients) supplemented by additional data from routine clinical practice (15 patients). Base and final model selection were based on the results of likelihood ratio tests. The model appropriateness was further assessed using numerical and graphical tools, as described below.

Based on previously published population pharmacokinetic models in malaria, RA, and systemic lupus erythematosus (SLE) and, on exploratory analysis, one-compartment and two-compartment structural models with first-order absorption and elimination were tested. The need for an absorption lag time parameter was tested.

Parameter estimation was performed in NONMEM using First-Order Conditional Estimation with the INTERACTION option.

Exponential models were used to describe the inter-individual variability (IIV) for the structural model parameters. Regardless of model form, IIV parameters was introduced and retained provided that estimates were neither very small nor caused instability in the model-fitting procedure.

Proportional and combined additive and proportional models were investigated to describe the residual variability. The residual variability model was developed until a smoothing spline of the absolute individual weighted residuals versus individual predictions was sufficient without trends.

Comparison between various potential models was based on a likelihood ratio test using the difference in the NONMEM objective function value ( $\triangle \mathrm{OFV}$ ) for two hierarchical competing models and where the number of degrees of freedom is equal to the difference in parameter numbers between the two models.

The covariate model development for HCQ was performed using the dataset. The covariates that were to be tested were: age, body weight, and sex.

The covariate model building was performed using stepwise covariate modeling: a forward inclusion phase and a backward elimination phase. In each step of the forward phase, covariate-parameter relationships were tested one at a time. The most significant relationship, given that it is 
statistically significant, was retained in the next step. Each remaining covariate-parameter relationship was again added one at a time to the model and the most significant was retained. This process was repeated until no more relationships were significant, at which point the full model was established. The full model was subsequently subjected to a stepwise backward elimination of relationships. Each covariate-parameter relationship was omitted from the model one at a time. The least significant relationship, given that it is not statistically significant, was removed from the model. This was repeated until no more relationships could be removed, at which point the final model was established. For the forward selection step, a $p<0.05\left(\chi_{\mathrm{p}=0.01, v=1}^{2}=3.84\right)$ was used while the backwards deletion step used a $p<0.001$ $\left(\chi_{\mathrm{p}=0.01, v=1}^{2}=10.83\right)$.

An allometric scaling model was used to describe the influence of body size and age on the clearance and volume parameters using Eq. 1:

$P_{i}=\theta_{1} \times\left(\frac{\mathrm{COV}_{i}}{\operatorname{COV}_{\mathrm{POP}}}\right)^{\theta_{2}} \times e^{\eta_{i}}$

where $\theta_{1}$ is the population value of the estimated parameter, $P_{i}$ is the individual-specific realization for the $i$ th subject with the value of the covariate $\left(\mathrm{COV}_{i}\right)$ scaled to a population typical value (), $\theta_{2}$ is the allometric effect and $\eta_{i}$ is the individual-specific realization of a random variable $\eta \sim \mathcal{N}\left(0, \omega^{2}\right)$ (IIV).

A linear model was used to describe the influence of sex on the clearance and volume parameters.

The final model will contain estimates for the model parameters and their associated IIV, and estimates for the residual variability and covariate effects. The criteria for accepting the NONMEM model estimation as the final run included the following:

- A 'successful minimization' statement issued by the NONMEM program, indicating convergence of maximum likelihood estimation.

- The standard error of estimates (if any) should preferably be less than half the estimate itself.

- There should be no unacceptable trends in the basic goodness-of-fit plots.

In order to provide an additional assessment of parameter uncertainty not relying on normality assumptions, the final model was bootstrapped 300 times.

Model evaluation included graphical [i.e., goodnessof-fit plots, prediction-corrected visual predictive checks (pcVPCs), normalized prediction distribution errors (NPDEs)] and numerical (bootstraps) evaluation.
External validation was performed by overlapping model predictions with additional data from 8 patients as part of routine clinical practice.

\subsection{Simulation Approaches to Inform Dose Optimization}

The obtained final POPPK model was used to discuss whether the dosing strategies currently applied are adequate in producing HCQ concentration profiles consistent with the concentration ranges in responders to treatment. Simulations were used to compare the exposure ranges with alternative dosing regimens.

\section{Results}

A total of 84 concentration samples were obtained from the 33 patients enrolled in the clinical study and from the additional 23 patients treated for COVID-19 disease in St Luc and St-Pierre Hospitals. None of the concentrations measured were above or below the limit of quantification. An exploratory plot of the observed concentrations is shown in Supplementary Figure S1. Body weight values were missing for 2 patients. They were replaced by the median value in the initial dataset. The remaining patients were used for external validation. Characteristics of patients included the model building, and validation datasets are described in Table 1, together with their baseline clinical symptoms and viral load. A one-compartment model with first-order absorption and elimination best described the observed data. The addition of a lag time component did not improve the model fitting performance, so this parameter was therefore not kept in the model for subsequent steps. Correlation was visually tested between candidate covariates, and the results are shown in Fig. 1. Good correlation was found between body weight and sex. Age was not correlated with body weight or sex.

During the covariate model building, body weight, and sex were found to significantly influence the clearance, with a higher drop in the objective function value for the body weight effect. However, because of the correlation between both, only body weight was kept in the final model. The influence of age on clearance did not reach the predefined statistical significance level to be kept in the final model. The final POPPK model was therefore a one-compartment model with the body weight effect on clearance. Final model parameter estimates are summarized in Table 2, which also includes bootstrap results and corresponding parameters in the previously published model in RA [2], for easy comparison. All the parameters were plausible given the data, and adequately estimated as also supported by the bootstrap results. The model adequately fitted the observed data, as 
Table 1 Summary baseline characteristics of patients included in the model building and external validation datasets

\begin{tabular}{|c|c|c|c|c|c|c|}
\hline Demographic and clinical data & $\begin{array}{l}\text { Model building } \\
\text { dataset }(n=48)\end{array}$ & $\begin{array}{l}\text { Number of } \\
\text { missing data }\end{array}$ & $\begin{array}{l}\text { External validation } \\
\text { dataset }(n=8)\end{array}$ & $\begin{array}{l}\text { Number of } \\
\text { missing data }\end{array}$ & Total $(n=56)$ & $\begin{array}{l}\text { Number } \\
\text { of missing } \\
\text { data }\end{array}$ \\
\hline Sex (males/females) & $26 / 22$ & & $5 / 3$ & & $31 / 25$ & \\
\hline $\begin{array}{l}\text { Weight }(\mathrm{kg}) \\
\text { Median [min; max] }\end{array}$ & $80[50,122]$ & 2 & $86.5[52 ; 108]$ & & $81.5[50 ; 122]$ & 2 \\
\hline $\begin{array}{l}\text { Age (years) } \\
\text { Median [min; max] }\end{array}$ & $58.5[21 ; 93]$ & & $57.5[38 ; 75]$ & & $58.5[21 ; 93]$ & \\
\hline Fever/chills $(\mathrm{y} / \mathrm{n})$ & $29 / 19$ & & $7 / 1$ & & $36 / 20$ & \\
\hline Cough $(y / n)$ & $25 / 23$ & & $7 / 1$ & & $32 / 24$ & \\
\hline Dyspnea (y/n) & $21 / 27$ & & $5 / 3$ & & $26 / 30$ & \\
\hline Conjunctivitis $(\mathrm{y} / \mathrm{n})$ & $0 / 48$ & & $0 / 8$ & & $0 / 56$ & \\
\hline Sore throat $(y / n)$ & $9 / 39$ & & $1 / 7$ & & $10 / 46$ & \\
\hline Nasal discharge $(\mathrm{y} / \mathrm{n})$ & $8 / 40$ & & $3 / 5$ & & $11 / 45$ & \\
\hline Headache $(y / n)$ & $12 / 36$ & & $2 / 6$ & & $14 / 42$ & \\
\hline General weakness $(y / n)$ & $23 / 24$ & 1 & $5 / 3$ & & $28 / 27$ & 1 \\
\hline Myalgia (y/n) & $16 / 31$ & 1 & $4 / 4$ & & $20 / 35$ & 1 \\
\hline Nausea/vomiting (y/n) & $4 / 44$ & & $4 / 4$ & & $8 / 48$ & \\
\hline Diarrhea $(y / n)$ & $8 / 40$ & & $3 / 5$ & & $11 / 45$ & \\
\hline Anosmia $(y / n)$ & $4 / 43$ & 1 & $0 / 1$ & 7 & $4 / 44$ & 8 \\
\hline Agueusia (y/n) & $6 / 42$ & & $0 / 1$ & 7 & $6 / 43$ & 7 \\
\hline Cardiovascular pathology $(\mathrm{y} / \mathrm{n})$ & $20 / 28$ & & $4 / 4$ & & $24 / 32$ & \\
\hline Hypertension (y/n) & $18 / 30$ & & $4 / 4$ & & $22 / 34$ & \\
\hline $\begin{array}{l}\mathrm{CRP}(\mathrm{mg} / \mathrm{dl}) \\
\text { Median [min; max] }\end{array}$ & $69.4[3.2,231.4]$ & & $57.4[22.5,190.3]$ & & $67.9[3.2,231.4]$ & \\
\hline $\begin{array}{l}\text { White blood cells }\left(1000 / \mathrm{mm}^{3}\right) \\
\text { Median }[\min ; \max ]\end{array}$ & $5.88[3.14,13.62]$ & 2 & $4.73[2.55,10.01]$ & & $5.76[2.55,13.62]$ & 2 \\
\hline $\begin{array}{l}\text { Lymphocytes } \\
\left(1000 / \mathrm{mm}^{3}\right) \\
\text { Median }[\mathrm{min} ; \max ]\end{array}$ & $0.9[0.19,2.13]$ & & $0.94[0.31,1.57]$ & & $0.90[0.19,2.13]$ & \\
\hline $\begin{array}{l}\mathrm{LDH}(\mathrm{U} / \mathrm{L}) \\
\text { Median [min; max] }\end{array}$ & $313[162,2555]$ & 1 & $333[296,429]$ & & $316[162,2555]$ & 1 \\
\hline $\begin{array}{l}\text { AST (U/L) } \\
\text { Median [min; max] }\end{array}$ & $32[13,968]$ & 2 & $34.5[14,46]$ & & $32.5[13,968]$ & 2 \\
\hline $\begin{array}{l}\text { ALT (U/L) } \\
\text { Median [min; max] }\end{array}$ & $28.5[8,604]$ & 2 & $30[13,42]$ & & $28.5[8,604]$ & 2 \\
\hline $\begin{array}{l}\text { Creatinine }(\mathrm{mg} / \mathrm{dl}) \\
\text { Median [min; max] }\end{array}$ & $1.02[0.55,2.77]$ & & $1.13[0.62,3.33]$ & & $1.04[0.55,3.33]$ & \\
\hline $\begin{array}{l}\text { Hemoglobin }(\mathrm{mg} / \mathrm{dl}) \\
\text { Median [min; } \max ]\end{array}$ & $13.2[9.4,16.5]$ & 2 & $13.1[8.9,16.2]$ & & $13.2[8.9,16.5]$ & 2 \\
\hline $\begin{array}{l}\text { Saturation }(\%) \\
\text { Median [min; } \max ]\end{array}$ & $95[83,98]$ & 1 & $92.5[84,100]$ & & $95[83,100]$ & 1 \\
\hline
\end{tabular}

shown by the basic goodness-of-fit plots (Fig. 2a-d), the NPDEs plots (Fig. 2e-f), and the pcVPCs based on 1000 simulations of the used dataset and with binning on time (Fig. 3). Weight influences the clearance value through an allometric relationship. Figure 4 shows the post hoc clearance values with respect to the patient body weights.

This model was used to simulate different dosing scenarios (for 5 and 10 days) as implemented in clinical study protocols and national guidelines. The results of the different simulations are shown in Fig. 5, and related probability of target attainment based on in vitro vero cells EC50 are provided in Table $\mathrm{S} 1$.

Figure 6 shows the predictions obtained with the model by Carmichael et al. [2] using the dosing regimen of $400 \mathrm{mg}$ twice daily (BID) on day 1, followed by $200 \mathrm{mg}$ BID the subsequent days (Belgian protocol). The observed concentrations in our model building dataset are overlaid. 

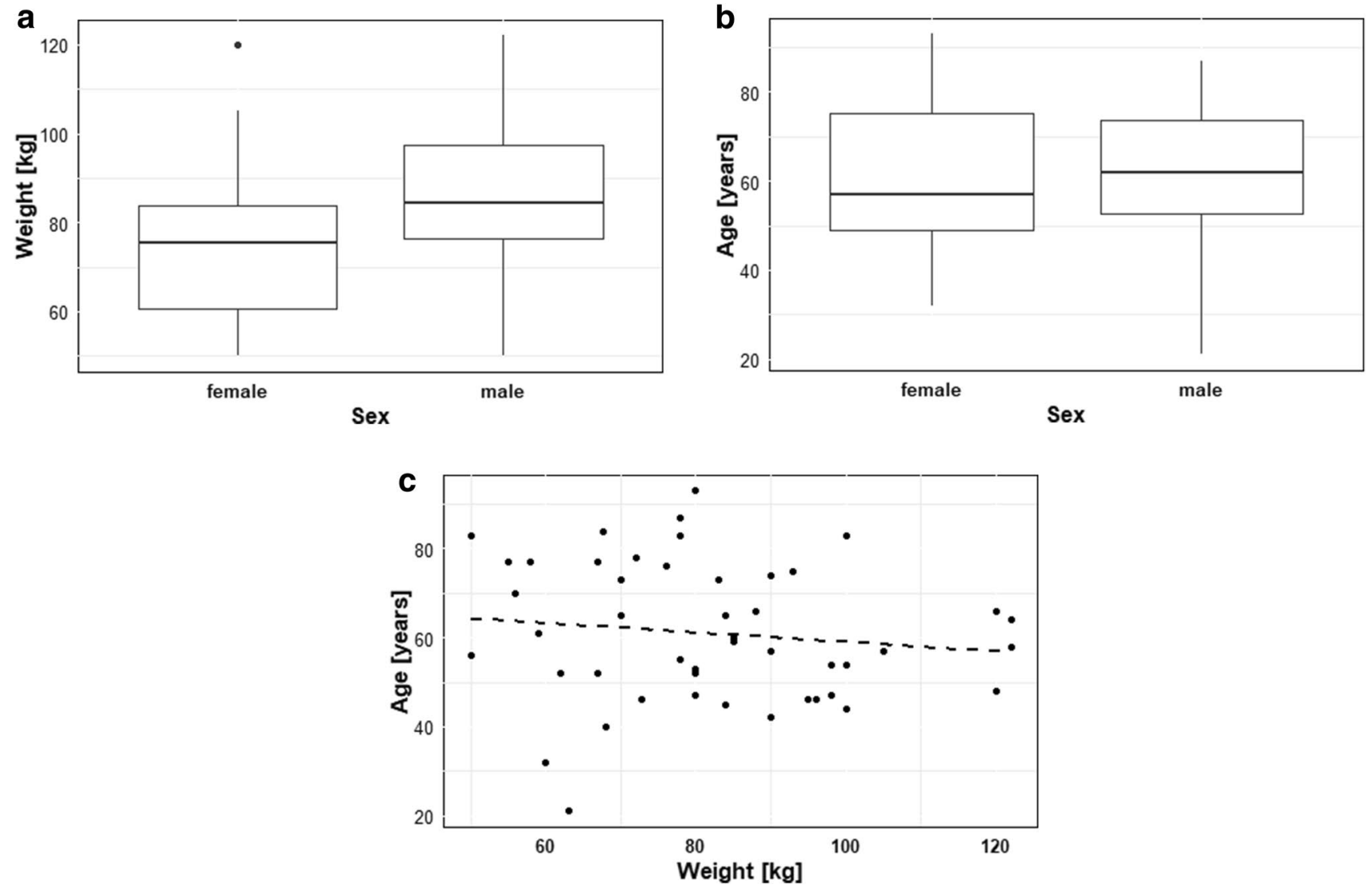

Fig. 1 Visual inspection of correlation between the patient characteristics: a boxplot of weight vs. sex, $\mathbf{b}$ boxplot age vs. sex, $\mathbf{c}$ age vs. weight. In the boxplots, the ends of the box are the 1st and the 3rd quartiles (comprise $50 \%$ of the observations), the central line represents the median and the whiskers correspond to 1.5 times the interquartile range (dots are the outliers)
Table 2 Final model parameter estimates of hydrochloroquine

\begin{tabular}{lllll}
\hline Parameter & Estimate & $\begin{array}{l}\text { Bootstrap results } \\
\text { Median }(95 \% \mathrm{CI})\end{array}$ & Shrinkage (\%) & $\begin{array}{l}\text { Corresponding estimated value in } \\
\text { model by Carmichael et al. [2] }\end{array}$ \\
\hline Ka $(/ \mathrm{h})$ & 9.3 & $7.31(1.81-9.44)$ & - & 0.765 \\
$V(\mathrm{~L})$ & 860.8 & $869.3(679-1130)$ & - & 605 \\
$\mathrm{CL}(\mathrm{L} / \mathrm{h})$ & 15.7 & $15.4(12.1-17.6)$ & - & 9.89 \\
$F$ & 0.746 (fixed) & & - & 0.746 \\
Tlag $(\mathrm{h})$ & ne & & & 0.445 \\
WT effect on CL & 1.38 & $1.39(0.56-2.52)$ & - & - \\
IIV on Ka $\left(\omega^{2}\right)$ & ne & & & 0.94 \\
IIV on CL $\left(\omega^{2}\right)$ & 0.15 & $0.13(0.04,0.21)$ & 23.3 & 0.127 \\
IIV on V $\left(\omega^{2}\right)$ & 0.27 & $0.26(0.13,0.38)$ & 27.6 & 0.25 \\
IIV on F $\left(\omega^{2}\right)$ & ne & & & 0.004 \\
IIV on Tlag $\left(\omega^{2}\right)$ & ne & & & ne \\
Eprop $\left(\sigma^{2}\right)$ & 0.029 & $0.028(0.01,0.045)$ & 40.7 & 0.044 \\
Eadd $\left(\sigma^{2}\right)$ & ne & & & 0.000365
\end{tabular}

$C L$ clearance, Eadd additional error, Eprop proportional error, $F$ bioavailability, IIV interindividual variability, $K a$ absorption rate constant, $n e$ not estimated, Tlag lag time, $V$ volume of distribution 

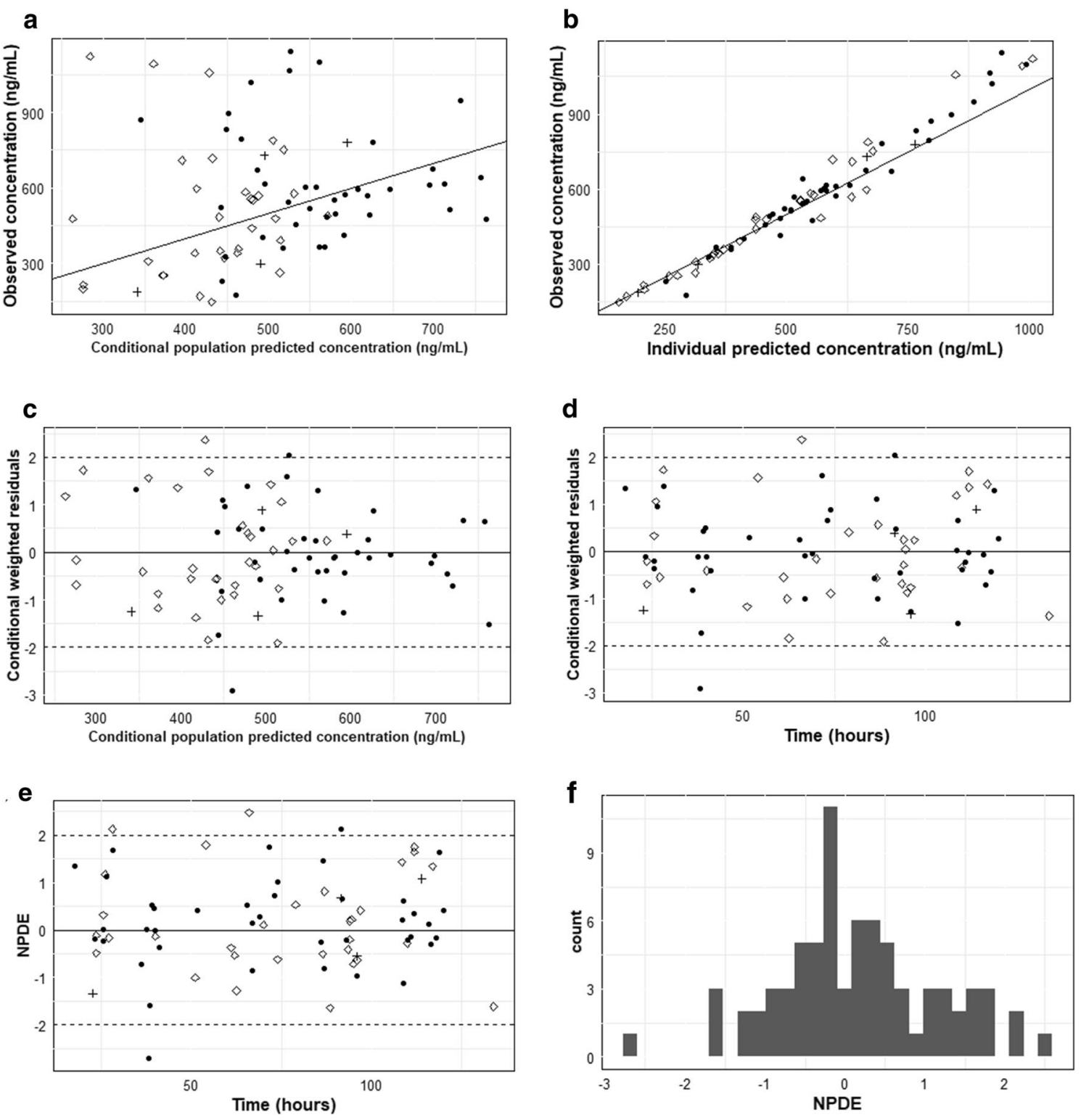

Fig. 2 Basic goodness-of-fit plots and normalized prediction distribution errors (NPDEs) plots: a observed hydrochloroquine concentration vs. population prediction, $\mathbf{b}$ observed concentration vs. Individual prediction, $\mathbf{c}$ residuals vs. population prediction, $\mathbf{d}$ residuals

\section{Discussion}

Six POPPK models are available in the literature for HCQ. $[1-3,20,21]$ Two of these were developed using wholeblood HCQ concentrations in patients with RA [2] and CLE [3]. The four additional models describe plasma concentrations, or merged blood and plasma concentrations, in different indications [1, 3, 21, 22]. The estimated apparent clearance values were quite consistent across previously published POPPK models: 10-14 L/h when whole-blood concentrations were analyzed $[1,2]$ versus $11-68 \mathrm{~L} / \mathrm{h}$ when

vs. time, e NPDEs vs. TIME, $\mathbf{f}$ histogram of NPDEs. Filled circles, open squares, and crosses correspond to patients with WT $\leq 80 \mathrm{~kg}$, WT $>80 \mathrm{~kg}$, and missing WT, respectively. Solid lines are either the line of identity $(\mathbf{a}, \mathbf{b})$ or the line $x=0(\mathbf{c}-\mathbf{e})$

plasma concentrations were analyzed [1, 3, 21, 22]. We are hereby reporting for the first time a POPPK model in COVID-19 patients. The fitting of model by Carmichael et al. [2] without parameter re-estimation resulted in an over-estimation of the concentrations observed in our study (see Fig. 6). This may be due to the fact that, unlike the previously published models, a strong effect of body weight was found on clearance in our final model (see Table 2). The predictive performances of our model were confirmed on an external set of data collected as part of standard of care and not used for model building (the result is shown 


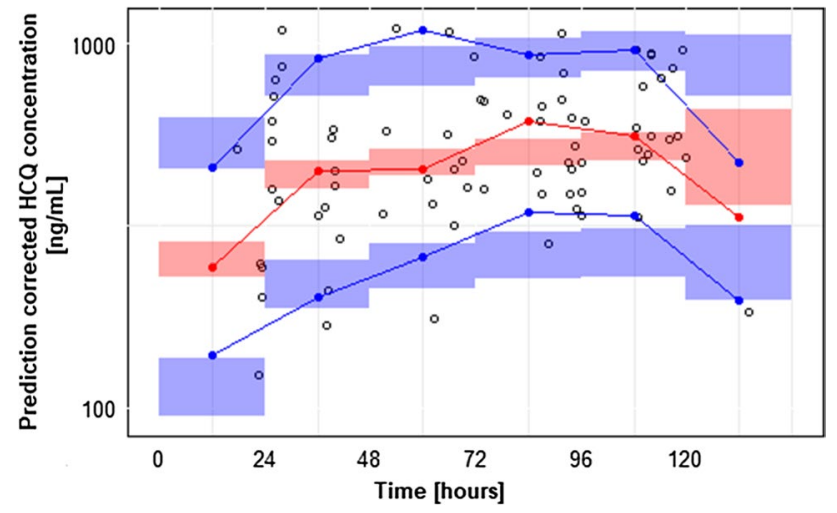

Fig. 3 Prediction-corrected VPC (1000 simulations of the dataset) with binning on time. Width of each bin is $24 \mathrm{~h}$. Red and blue lines are the median and the 5th and 95th percentiles of the predictioncorrected observed blood concentration of hydrochloroquine (HCQ) in each bin. Red and blue areas are the simulation-based 90\% confidence interval for the model predicted median and 5th and 95th percentiles of each bin. Points represent the prediction-corrected observations

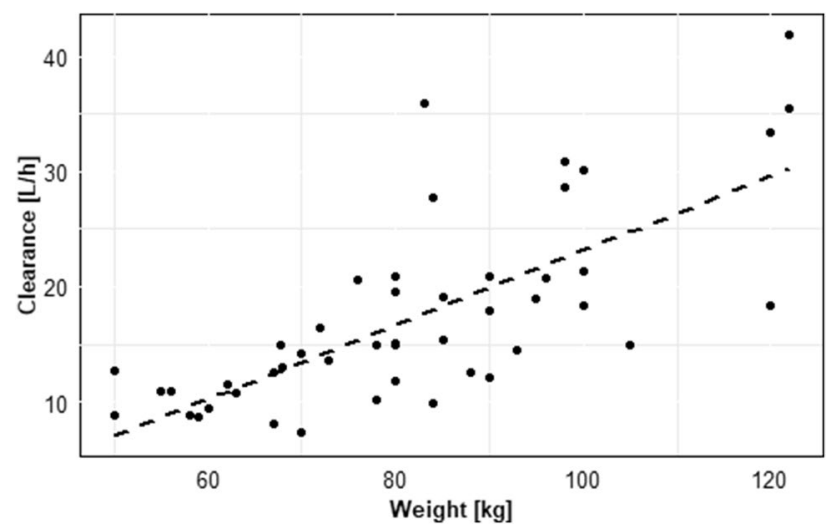

Fig. 4 Individual-specific hydrochloroquine clearance parameter value (post hoc) vs. body weight

in Supplementary Figure S2). It should, however, be noted that this model still carries a moderate to high unexplained variability component on the volume of distribution and clearance parameters, which can be explained by untested covariates, such as concomitant use of CYP2D6 modulators and underlying renal impairment. Another difference from previously published models is the higher estimate of $\mathrm{Ka}$ in our model. This value can be explained by tablet crunching and administration through nasogastric tubes in some of the patients who were in ICU or unable to swallow the tablets. However, this information was not consistently collected and could therefore not be adequately tested during the covariate analysis.
The actual value of the present model resides in its ability to inform the characterization of reference concentrations for efficacy and/or safety of HCQ in COVID-19, and therefore informs the characterization of the therapeutic range and the (range of) safe and effective doses. For this, there is a need for an exposure-response rationale regarding drug efficacy and drug safety. Good understanding of the determinants of clinical efficacy and safety (QT prolongation) would be the next step.

The mechanism of action of HCQ in the context of SARS-CoV-2 remains poorly defined. Although hydroxychloroquine's effect on direct inhibition of the virus and its anti-inflammatory and immunomodulatory activities are known, whether these mechanisms play a role against COVID-19 remains to be determined. [24].

While the application of HCQ to COVID-19 stems is justified by its past use as antiviral, including for SARSCoV-1, there has been conflicting evidence on HCQ effects in reducing viral load with different half-maximal effective concentration (EC50) or $90 \%$ of the maximal effective concentration (EC90) values reported [4, 5]. Moreover, according to the recent paper by Maisonnasse et al. [7], HCQ showed antiviral activity in African green monkey kidney cells (VeroE6) but not in a model of reconstituted human airway epithelium, and not in macaques after different treatment strategies were tested in comparison to placebo, before and after peak viral load, alone, or in combination with azithromycin. If their clinical relevance is established, reported EC50 and/or EC90 values can be used for in vitro and in vivo extrapolation to inform the dose selection in conjunction with the pharmacokinetic information provided by our model. For graphical and numerical comparative purposes, EC50 values by Yao et al. [4] $(0.72 \mu \mathrm{M})$ and Liu et al. [5] $(4.51 \mu \mathrm{M})$, scaled to whole-blood concentrations (assuming $50 \%$ protein binding in patients [25] and a serum/whole-blood ratio of 0.53 [26]), are included in Fig. 5. Information about the proportion of simulated subjects achieving target concentrations are provided in Supplementary Table S1. It should be noted that, if such an exercise is implemented, the dosing regimen that would lead to an average free minimum $\left(C_{\text {trough }}\right)$ plasma concentration higher than the reported in vitro EC50 or EC90 would be higher than the currently tested doses in most of clinical protocols and national guidelines so far published. This would have a particular impact in over-weight patients who would need higher doses due to body weight effects on clearance (see Fig. 4). One should, however, keep in mind that an immunomodulatory effect of HCQ in COVID-19 disease cannot be excluded. Similar to its effects in RA and immunological diseases, HCQ is supposed to have some effect on 

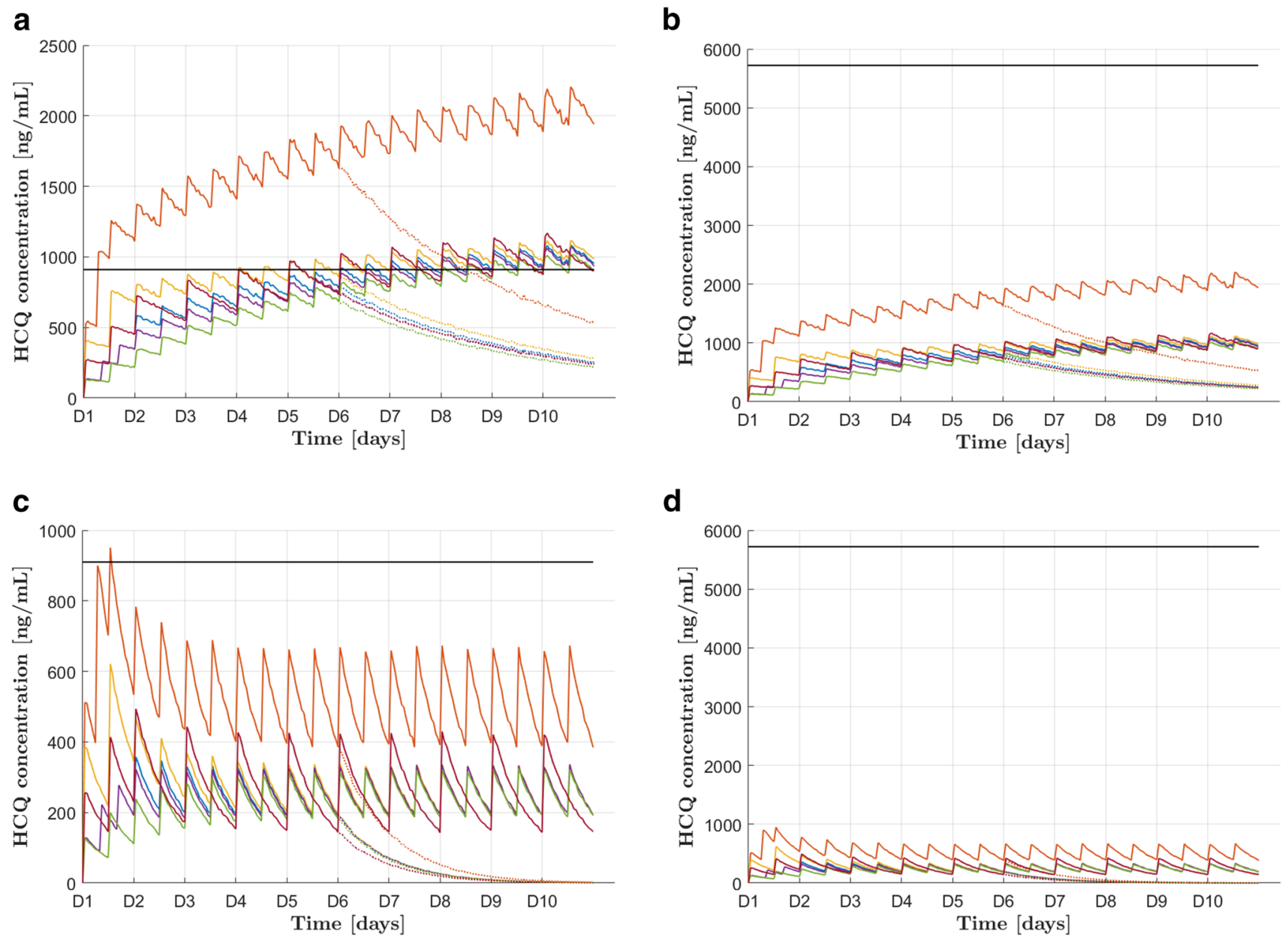

Fig. 5 Simulations of hydrochloroquine (HCQ) blood concentrations $\left(n_{\text {sim }}=1000\right)$ using the final model for two representative patients of $50 \mathrm{~kg}(\mathbf{a}, \mathbf{b})$ and $150 \mathrm{~kg}(\mathbf{c}, \mathbf{d})$. The horizontal line represents the projected target total whole blood concentrations based on EC50 values from Yao et al. [4] (a, c) and Liu et al. 5 (b, d). Blue curve median pharmacokinetic profile simulated according to the Belgian protocol, i.e., 400 twice daily (BID) on day 1, $200 \mathrm{mg}$ BID the subsequent days. Orange curve median pharmacokinetic profile simulated according to a dosing regimen of $800 \mathrm{mg}$ followed by $800 \mathrm{mg}$ $6 \mathrm{~h}$ later and $400 \mathrm{mg} 6 \mathrm{~h}$ later on day 1, $400 \mathrm{mg}$ BID the subsequent

immune activation. It has been described that HCQ may inhibit production of pro-inflammatory cytokines, [24] and therefore decrease the later inflammatory stage of the disease. An additive (or synergistic) anti-inflammatory effect could thereby support the need for doses lower than those determined based only on the potential antiviral effects.

As regards drug safety, matched concentration and QT data in the same patients are needed to develop a concentration-QT model that could be used in conjunction with

days. Yellow curve median pharmacokinetic profile simulated according to a dosing regimen of $600 \mathrm{mg}$ BID on day 1, $200 \mathrm{mg}$ BID the subsequent days. Purple curve median pharmacokinetic profile simulated according to a dosing regimen of $200 \mathrm{mg}$ TID on day $1,200 \mathrm{mg}$ BID the subsequent days. Green curve median pharmacokinetic profile simulated according to a dosing regimen of $200 \mathrm{mg}$ BID. Black red curve median pharmacokinetic profile simulated according to a dosing regimen of $400 \mathrm{BID}$ on day $1,400 \mathrm{mg}$ daily the subsequent days. Solid lines treatment for 10 days. Dotted lines treatment for 5 days

this model to characterize unsafe doses as regards QT prolongation.

A firm dose recommendation cannot be provided based solely on our pharmacokinetic results, given the remaining uncertainties in the characterization of the exposure-response for HCQ in COVID-19 for both efficacy and safety. Optimal dose selection should be informed by the pharmacokinetics and the exposure-response analyses. 


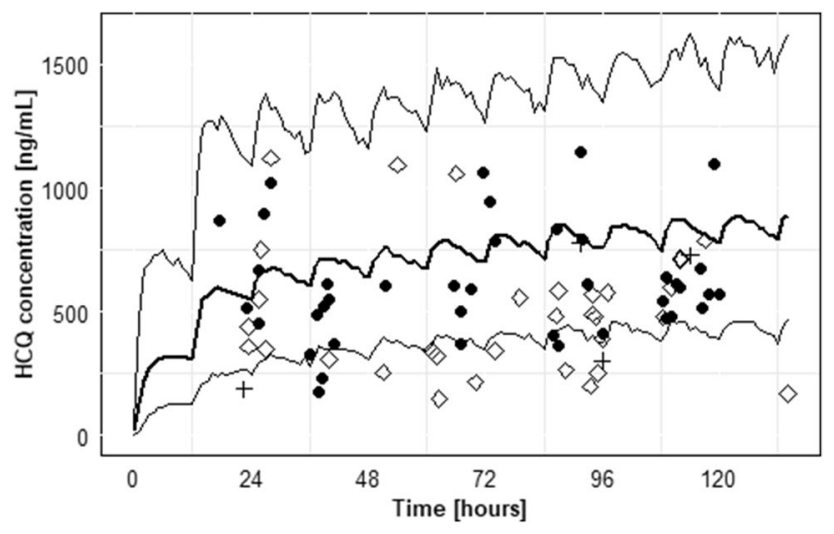

Fig. 6 Predictions of hydrochloroquine (HCQ) blood concentrations $\left(n_{\text {sim }}=1000\right)$ using the model of Carmichael et al. [1] Blue lines represent the median of the simulations and black lines are the 5th and 95th percentiles (90\% prediction interval). Points correspond to the observations in our study. Filled circles, open squares, and crosses correspond to patients with WT $\leq 80 \mathrm{~kg}$, WT $>80 \mathrm{~kg}$ and missing WT, respectively

Adequately conducted exposure-response analyses, essential to characterize the target concentrations for drug efficacy and safety, are currently lacking for HCQ in COVID19. When available, these would complement our findings and permit reliable dose recommendations for HCQ in COVID-19.

That said, some learning and clinical implications are already clear from our findings. The first is that, if one bases the dosing recommendation only on the in vitro results obtained with kidney cells (VeroE6), our simulation results show that the doses used in most of the protocols and clinical studies are suboptimal for HCQ efficacy. The second implication is that, given the important influence of body weight on drug clearance, this should be taken into account for dosing recommendations for HCQ in COVID-19 patients.

\section{Conclusion}

The developed models provided useful insight for the dosing optimization of HCQ in COVID-19 which is still an unmet and urgent need. The present results should be used in conjunction with exposure-efficacy and exposure-safety data to inform optimal dosing of HCQ in COVID-19.

\section{Declarations}

Funding No specific funding was received for the preparation of this article.
Conflict of Interest The authors declared no competing interest for this work.

Ethics approval The protocol (Eudract 2020-001434-35) was approved by the local ethical committee.

Consent to participate A written informed consent was obtained from each patient taking part to the study, in compliance with the International Council for Harmonisation (ICH) Good Clinical Practice (GCP) Guideline,

Consent for publication Patients expressed no objection for the publication of the results.

Availability of Data and Material (data transparency) The datasets generated during and/or analyzed during the current study are not publicly available due to confidentiality reasons but are available from the corresponding author on reasonable request.

Code Availability Custom NONMEM and R codes used for model building, simulations and computation/representation of pcVPC are available on the DDMoRe Model Repository http://repository.ddmor e.eu/model/DDMODEL00000322.

Author Contributions Not applicable.

\section{References}

1. Lim HS, Im JS, Cho JY, et al. Pharmacokinetics of hydroxychloroquine and its clinical implications in chemoprophylaxis against malaria caused by Plasmodium vivax. Antimicrob Agents Chemother. 2009;53(4):1468-75. https://doi.org/10.1128/aac.00339-08.

2. Carmichael SJ, Charles B, Tett SE. Population pharmacokinetics of hydroxychloroquine in patients with rheumatoid arthritis. Ther Drug Monit. 2003;25(6):671-81. https://doi.org/10.1097/00007 691-200312000-00005.

3. Morita S, Takahashi T, Yoshida Y, Yokota N. Population pharmacokinetics of hydroxychloroquine in Japanese patients with cutaneous or systemic lupus erythematosus. Ther Drug Monit. 2016;38:259-67. https://doi.org/10.1097/FTD.000000000000026 1.

4. Yao X, Ye F, Zhang M, et al. In vitro antiviral activity and projection of optimized dosing design of hydroxychloroquine for the treatment of severe acute respiratory syndrome coronavirus 2 (SARS-CoV-2). Clin Infect Dis. 2020. https://doi.org/10.1093/ $\mathrm{cid} / \mathrm{ciaa} 237$.

5. Liu J, Cao R, Xu M, et al. Hydroxychloroquine, a less toxic derivative of chloroquine, is effective in inhibiting SARS-CoV-2 infection in vitro. Cell Discov. 2020;6:16. https://doi.org/10.1038/ s41421-020-0156-0.

6. Wang M, Cao R, Zhang L, et al. Remdesivir and chloroquine effectively inhibit the recently emerged novel coronavirus (2019-nCoV) in vitro. Cell Res. 2020;30(3):269-71. https://doi. org/10.1038/s41422-020-0282-0.

7. Maisonnasse P, Guedj J, Contreras V, et al. Hydroxychloroquine use against SARS-CoV-2 infection in non-human primates. Nature. 2020. https://doi.org/10.1038/s41586-020-2558-4.

8. Gautret P, Lagierac JC, Parola P, et al. Hydroxychloroquine and azithromycin as a treatment of COVID-19: results of an openlabel non-randomized clinical trial. Int J Antimicrob Agents. 2020. https://doi.org/10.1016/j.ijantimicag.2020.105949. 
9. Gautret P, Lagier JC, Parola P, et al. Clinical and microbiological effect of a combination of hydroxychloroquine and azithromycin in 80 COVID-19 patients with at least a six-day follow up: A pilot observational study. Travel Med Infect Dis. 2020;34:101663. https ://doi.org/10.1016/j.tmaid.2020.101663.

10. Sciensano. Interim Clinical Guidance for adults with suspected or confirmed Covid-19 in Belgium. 31 March 2020; Version 6. https ://epidemio.wiv-isp.be/ID/Documents/Covid19/COVID19_Inter imGuidelines_Treatment_ENG.pdf. Accessed 17 Apr 2020.

11. Chen J, Liu D, Lui L, et al. A pilot study of hydroxychloroquine in treatment of patients with common coronavirus disease-19 COVID-19. J Zhejiang Univ. 2020;49(2):215-9.

12. Zhaowei C, Jijia H, Zongwei Z, et al. Efficacy of hydroxychloroquine in patients with COVID-19: results of a randomized clinical trial. medRxiv. 2020. https://doi.org/10.1101/2020.03.22.20040 758 .

13. Geleris J, Sun Y, Platt J, et al. Observational study of hydroxychloroquine in hospitalized patients with Covid-19. N Engl J Med. 2020. https://doi.org/10.1056/nejmoa2012410.

14. Catteau L, Dauby N, Montourcy M, et al. Low-dose hydroxychloroquine therapy and mortality in hospitalised patients with COVID-19: a nationwide observational study of 8075 participants. Int J Antimicrob Agents. 2020. https://doi.org/10.1016/j.ijantimica g.2020.106144.

15. World Health Organization. "Solidarity" clinical trial for COVID19 treatments. https://www.who.int/emergencies/diseases/novel -coronavirus-2019/global-research-on-novel-coronavirus-2019ncov/solidarity-clinical-trial-for-covid-19-treatments. Accessed 11 Sept 2020.

16. U.S. National Institutes of Health. Trial of Treatments for COVID19 in Hospitalized Adults (DisCoVeRy). In: ClinicalTrials.gov. https://clinicaltrials.gov/ct2/show/NCT04315948. Accessed 28 Aug 2020.

17. Horby P, Mafham M, et al. Effect of hydroxychloroquine in hospitalized patients with COVID-19: preliminary results from a multi-centre, randomized, controlled trial. medRxiv. https://doi. org/10.1101/2020.07.15.20151852

18. Martin-Blondel G, Ruiz S, Murris M, et al. Hydroxychloroquine in COVID-19 patients: what still needs to be known about the kinetics. Clin Infect Dis. 2020. https://doi.org/10.1093/cid/ciaa5 58.

19. Morrisette T, Lodise TP, Scheetz MH, et al. The pharmacokinetic and pharmacodynamic properties of hydroxychloroquine and dose selection for COVID-19: putting the cart before the horse. Infect Dis Ther. 2020. https://doi.org/10.1007/s40121-020-00325-2.

20. Thémans P, Dauby N, Schrooyen L, et al. Model informed dosing of hydroxycholoroquine in COVID-19 patients: learnings from the recent experience, remaining uncertainties and gaps. Br J Clin Pharmacol. 2020. https://doi.org/10.1111/bcp.14436.

21. Tett SE, Cutler DJ, Day RO, Brown KF, et al. Bioavailability of hydroxychloroquine tablets in healthy volunteers. Br J Clin Pharmacol. 1989;27(6):771-9. https://doi. org/10.1111/j.1365-2125.1989.tb03439.x.

22. Balevic SJ, Green TP, Clowse MEB, et al. Pharmacokinetics of hydroxychloroquine in pregnancies with rheumatic diseases. Clin Pharmacokinet. 2019;58(4):525-33. https://doi.org/10.1007/ s40262-018-0712-z.

23. Garcia-Cremades M, Solans BP, Hughes E, et al. Optimizing hydroxychloroquine dosing for patients with COVID-19: an integrative modeling approach for effective drug repurposing. Clin Pharmacol Ther. 2020;108(2):253-63. https://doi.org/10.1002/ cpt.1856.

24. Grassin-Delyle S, Salvator H, Brollo M, et al. Chloroquine inhibits the release of inflammatory cytokines by human lung explants. Clin Infect Dis. 2020. https://doi.org/10.1093/cid/ciaa546.

25. McLachlan AJ, Cutler DJ, Tett SE. Plasma protein binding of the enantiomers of hydroxychloroquine and metabolites. Eur J Clin Pharmacol. 1993;44(5):481-4. https://doi.org/10.1007/BF003 15548.

26. Blanchet B, Moez J, Allard M, et al. Hydroxychloroquine levels in patients with systemic lupus erythematosus: comparison of whole blood and serum levels [abstract]. Arthritis Rheumatol. 2019;71 (suppl 10). https://acrabstracts.org/abstract/hydroxychloroquinelevels-in-patients-with-systemic-lupus-erythematosus-comparison -of-whole-blood-and-serum-levels/. Accessed 20 Apr 2020.

\section{Affiliations}

\section{Pauline Thémans ${ }^{1}$ - Leila Belkhir ${ }^{2}$ - Nicolas Dauby ${ }^{3,4}$ - Jean-Cyr Yombi ${ }^{2}$. Julien De Greef ${ }^{2}$. Kevin-Alexandre Delongie ${ }^{5}$ Martin Vandeputte ${ }^{4} \cdot$ Rakan Nasreddine $^{3} \cdot$ Xavier Wittebole $^{2} \cdot$ Francoise Wuillaume $^{4}$. Cécile Lescrainier $^{6}$. Veerle Verlinden ${ }^{6}$. Sophie Kiridis ${ }^{6}$. Jean-Michel Dogné ${ }^{6,7}$. Jamila Hamdani ${ }^{6}$. Pierre Wallemacq ${ }^{5} \cdot$ Flora T. Musuamba $^{6,8} \mathbb{D}$}

$1 \quad$ Namur Institute for Complex Systems (naXys) and Department of Mathematics, University of Namur, Namur, Belgium

2 Department of Internal Medicine and Infectious Diseases, Cliniques Universitaires Saint Luc, Université Catholique de Louvain, Brussels, Belgium

3 Department of Infectious Diseases, Centre Hospitalier Saint-Pierre, Université Libre de Bruxelles (ULB), Brussels, Belgium

4 Environmental Health Research Centre, Public Health School, Université Libre de Bruxelles (ULB), Brussels, Belgium
5 Department of Clinical Chemistry, Cliniques Universitaires St Luc, Université catholique de Louvain, Brussels, Belgium

6 Belgian Federal Agency for Medicines and Health Products, 1060 Brussels, Belgium

7 Department of Pharmacy, Namur Thrombosis and Hemostasis Center, University of Namur, Namur, Belgium

8 Faculty of Pharmaceutical Sciences, University of Lubumbashi, Lubumbashi, Democratic Republic of the Congo 